\title{
A new Pareto-type distribution with applications in reliability and income data
}

\author{
Marcelo Bourguignon $^{1 *}$, Helton Saulo ${ }^{2}$ and Rodrigo Nobre Fernandez ${ }^{3}$ \\ ${ }^{1}$ Departamento de Estatística, Universidade Federal do Rio Grande do Norte, Natal, RN, Brazil \\ ${ }^{2}$ Instituto de Matemática e Estatística, Universidade Federal de Goiás, Goiânia, Brazil \\ ${ }^{3}$ Department of Economics, Universidade Federal de Pelotas, Pelotas, Brazil
}

\begin{abstract}
A new Pareto-type distribution is introduced and studied. This new model is a generalization of the well-known Pareto distribution. We derive some of its probabilistic and inferential properties. We deduce the mathematical form of the Lorenz curve and the Gini index associated with the new model. The maximum likelihood estimators are derived and their performance are evaluated through a Monte Carlo simulation study. Finally, we illustrate the flexibility of the new distribution by means of three applications to real data sets.
\end{abstract}

Keywords Pareto distribution; Income distribution; Maximum likelihood estimation; Gini coefficient; Reliability.

Mathematics subject classification Primary 33C90; Secondary 62E99.

\section{Introduction}

The Pareto distribution (Pareto [14]) was originally proposed to model the unequal distribution of wealth since he observed the way that a larger portion of the wealth of any society is owned by a smaller percentage of the people. The Pareto distribution plays an important role in analysing a wide range of real-world situations, not only in the field of economics. For example, in actuarial sciences, reliability, finance and climatology, where it usually describes the occurrence of extreme weather. Several alternative forms of the Pareto distribution can be found in the literature. Dragulescu and Yakovenko [5], Silva [15], Yakovenko and Rosser [17] advanced in an exponential distribution of individual income similar to the Boltzmann-Gibbs. Clementi et al. [2,3,4] proposed a generalized $\kappa$ distribution and Willis and Mimkes [16] used lognormal and Boltzmann distributions to argue for a separate treatment of employed and self-employed income. Moura Jr. and Ribeiro [12] and Figueira et al. [6] showed that the Gompertz curve combined with Pareto's law is a good descriptive model for income distribution.

In this context, this paper aims to introduce a new Pareto-type distribution that offers a better fit in certain practical situations (see Section 7). The new distribution is characterized by transformation

*Corresponding author. Email: m.p.bourguignon@gmail.com 
of the half logistic distribution. We provide a comprehensive account of the mathematical properties of the proposed distribution. It should be highlighted that, compared with the Pareto distribution, our model has the following advantages: (1) it can have an upside-down bathtub or a decreasing hazard rate function depending on the values of its parameters; (2) mathematical simplicity. For instance, the probability and distribution functions of the new distribution have a simple form in contrast with some generalizations of the Pareto distribution which have associated probability function involving special functions (log, beta and gamma functions); and (3) the proposed distribution has only two parameters, in contrast with some generalizations of the Pareto distribution which have three or four parameters.

This study is organized in eight sections starting with this introduction. In Sections 2 and 3, we present the new model and derive some of its properties. Explicit expressions for the Lorenz curve and Gini index are derived in Section 4. In Section 5, we discuss maximum likelihood (ML) estimation of the model parameters. We present a Monte Carlo (MC) simulation experiment to evaluate the ML estimates of the model parameters in Section 6. Three applications in Section 7 illustrate the usefulness of the new distribution for data modeling. Finally, we have the final considerations.

\section{The new model}

The cumulative distribution function (CDF) of the new Pareto-type distribution (NP) is given by

$$
F(x ; \alpha, \beta)=\frac{1-(\beta / x)^{\alpha}}{1+(\beta / x)^{\alpha}}=\frac{x^{\alpha}-\beta^{\alpha}}{x^{\alpha}+\beta^{\alpha}}=1-\frac{2(\beta / x)^{\alpha}}{1+(\beta / x)^{\alpha}}=1-\frac{2 \beta^{\alpha}}{x^{\alpha}+\beta^{\alpha}}, \quad x \geq \beta,
$$

where $\alpha>0$ is a shape parameter and $\beta>0$ is the scale parameter. In this case, the notation $X \sim \mathrm{NP}(\alpha, \beta)$ is used.

For high incomes this formula closely approximates the form

$$
F(x ; \alpha, \beta)=1-A x^{-\alpha},
$$

which is the form predicted by Pareto's law. The model specified in (1) possesses this important property of the weak Pareto law (Mandelbrot [10]), that is, (1) converges in distribution to the Pareto model for $x$ sufficiently large.

Lemma 1 (A characterization) If a random variable $(R V) Y$ follows the half logistic distribution with parameters $\alpha$, then the $R V X=\beta e^{Y}$ has the $C D F$ in Equation (1) with parameters $\alpha$ and $\beta$.

The representation above highlights the useful observation that the logarithm of such a variable has a shifted half logistic distribution. It will permit the recognition of many distributional properties of the new model as reflections of parallel properties of half logistic variables.

Remark 1 If $Z$ has the exponential distribution then $Y=\beta e^{Z}$ has the Pareto distribution.

Remark 2 If $Z$ has the logistic distribution then $Y=\beta e^{Z}$ has the log-logistic distribution. 
The probability density function (PDF) corresponding to (1) is

$$
f(x ; \alpha, \beta)=\frac{2 \alpha(\beta / x)^{\alpha}}{x\left[1+(\beta / x)^{\alpha}\right]^{2}}=\frac{2 \alpha(\beta / x)^{\alpha+1}}{\beta\left[1+(\beta / x)^{\alpha}\right]^{2}}=\frac{2 \alpha \beta^{\alpha} x^{\alpha-1}}{\left(x^{\alpha}+\beta^{\alpha}\right)^{2}}, \quad x \geq \beta .
$$

It can be shown that

$$
\lim _{x \rightarrow \beta} f(x ; \alpha, \beta)=\frac{\alpha}{2 \beta} \text { and } \lim _{x \rightarrow \infty} f(x ; \alpha, \beta)=0 .
$$

Proposition 1 For $x \geq \beta>0$, the NP density function is decreasing.

Proof:

The first derivative of $f(x ; \alpha, \beta)$ becomes

$$
f^{\prime}(x ; \alpha, \beta)=\frac{\mathrm{d} f(x ; \alpha, \beta)]}{\mathrm{d} x}=\frac{2 \alpha \beta^{a} x^{\alpha-2}}{\left(x^{\alpha}+\beta^{\alpha}\right)^{3}} \Delta(x),
$$

where $\Delta(x)=(\alpha-1) \beta^{\alpha}-(\alpha+1) x^{\alpha}$. Clearly, $\Delta(x)$ is strictly decreasing in $x$ with $\Delta(\beta)=-2 \beta^{\alpha}$ and $\Delta(\infty)=-\infty$.

Remark 3 The density function of $X$ can be expressed as

$$
f(x ; \alpha, \beta)=\frac{2}{\left[1+(\beta / x)^{\alpha}\right]^{2}} \cdot g(x ; \alpha, \beta),
$$

where $g(x ; \alpha, \beta)=\alpha \beta^{\alpha} x^{-(\alpha+1)}$ is the PDF of the Pareto distribution. The multiplying quantity $2 /\left[1+(\beta / x)^{\alpha}\right]^{2}$ works as a corrected factor for the PDF of the Pareto model.

From Remark 3 , note that $f(x ; \alpha, \beta) \geq g(x ; \alpha, \beta)$ for $x \geq \beta(\sqrt{2}-1)^{-1 / \alpha}$ and $f(x ; \alpha, \beta)<$ $g(x ; \alpha, \beta)$ for $x<\beta(\sqrt{2}-1)^{-1 / \alpha}$. Figure 1 illustrates its PDF shapes for some parameter values.

From Equation (2), we observe that

$$
f(x ; \alpha, \beta)=\frac{\alpha}{x}\left\{F(x ; \alpha, \beta)[1-F(x ; \alpha, \beta)]+\frac{1}{2}[1-F(x ; \alpha, \beta)]^{2}\right\},
$$

or equivalently

$$
f(x ; \alpha, \beta)=\frac{\alpha}{x}\left\{[1-F(x ; \alpha, \beta)]-\frac{1}{2}[1-F(x ; \alpha, \beta)]^{2}\right\} .
$$

Furthermore, note that

$$
F(x ; \alpha, \beta)=\int_{0}^{-\log (\beta / x)} \frac{2 \alpha \mathrm{e}^{-\alpha t}}{\left(1+\mathrm{e}^{-\alpha t}\right)^{2}} d t .
$$

The quantile function corresponding to (1) is

$$
Q(u)=F^{-1}(u)=\beta\left(\frac{1+u}{1-u}\right)^{1 / \alpha}, 0<u<1 .
$$




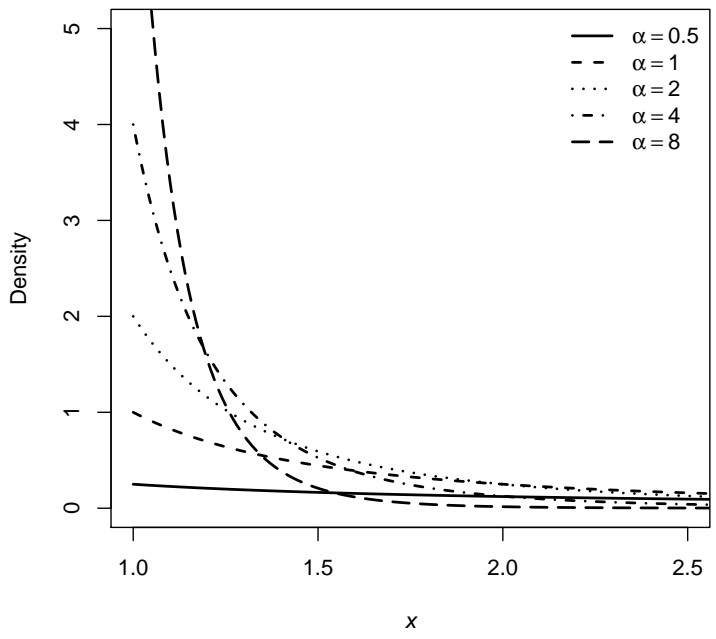

(a)

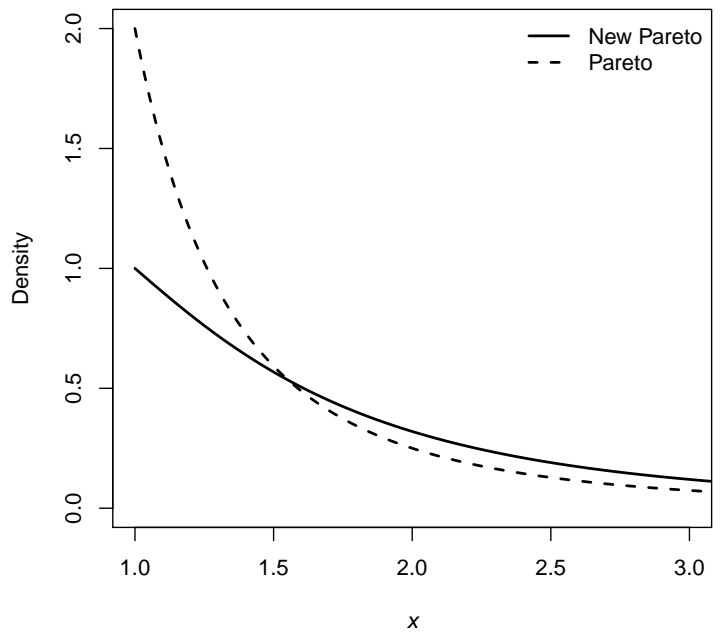

(b) $\alpha=2$

Figure 1: The NP density function for some parameter values; $\beta=1$.

In particular, the median is given by

$$
\operatorname{median}(X)=\beta 3^{1 / \alpha},
$$

and the median of the Pareto distribution is given by $\beta 2^{1 / \alpha}<\beta 3^{1 / \alpha}$.

\section{Properties of the new model}

\subsection{Hazard rate function}

The corresponding hazard rate (HR) is

$$
h(x ; \alpha, \beta)=\frac{\alpha}{x\left[1+(\beta / x)^{\alpha}\right]}=\frac{\alpha x^{\alpha-1}}{x^{\alpha}+\beta^{\alpha}} .
$$

It can be shown that

$$
\lim _{x \rightarrow \beta} h(x ; \alpha, \beta)=\frac{\alpha}{2 \beta} \text { and } \lim _{x \rightarrow \infty} h(x ; \alpha, \beta)=0 .
$$

Proposition 2 For $\beta>0$, the NP distribution has an decreasing $H R$ if $\alpha \leq 1$, and it has a unimodal $H R$ if $\alpha>1$. 
Proof:

The first derivative of $h(x ; \alpha, \beta)$ becomes

$$
h^{\prime}(x ; \alpha, \beta)=\frac{\mathrm{d} h(x ; \alpha, \beta)]}{\mathrm{d} x}=\frac{\alpha x^{\alpha-2}\left[(\alpha-1) \beta^{\alpha}-x^{\alpha}\right]}{\left(x^{\alpha}+\beta^{\alpha}\right)^{2}} .
$$

For $\alpha \leq 1$, it is clear that $h^{\prime}(x ; \alpha, \beta)<0, h(x ; \alpha, \beta)$ is decreasing in $x$. For $\alpha>1$, the function $h(x ; \alpha, \beta)$ has a unique mode at $x=x_{0}$ with $h(x ; \alpha, \beta)$ increasing for all $x<x_{0}$ and decreasing for all $x>x_{0}$, where $x_{0}=\beta(\alpha-1)^{1 / \alpha}$.

Note that $h(x ; \alpha, \beta) \leq h_{P}(x ; \alpha, \beta)$ for all $x \geq \beta$, where $h_{P}(x ; \alpha, \beta)=\alpha / x$ is the HR of the Pareto distribution. Figure 2 displays the behavior of $h(x ; \alpha, \beta)$ for selected values of the model parameters.

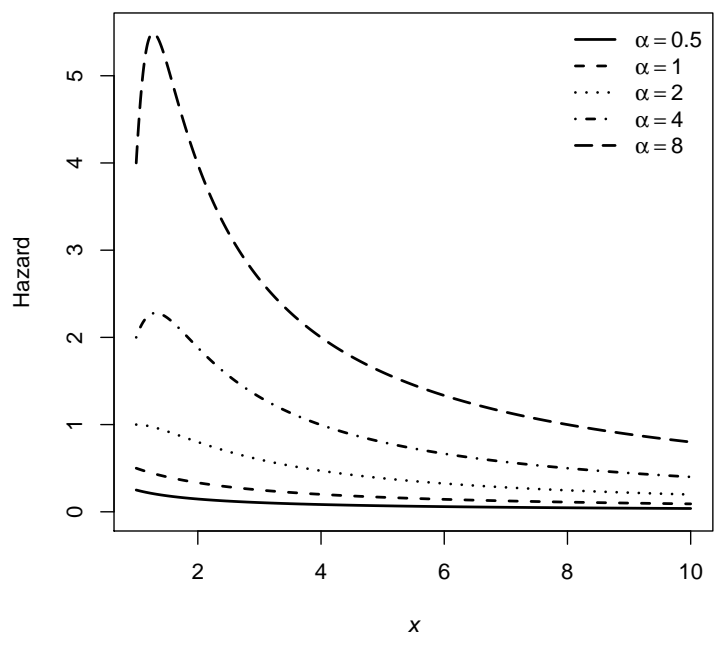

(a)

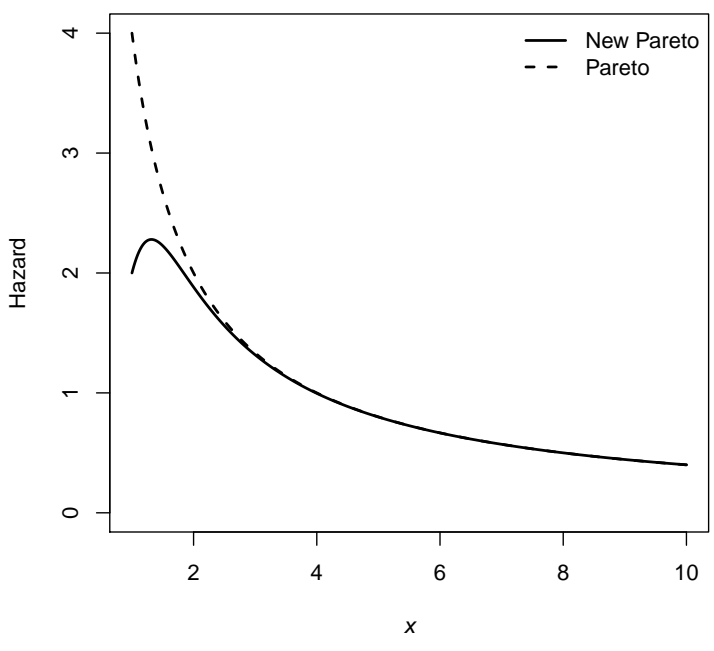

(b) $\alpha=4$

Figure 2: Plots of the NP hazard function for some parameter values; $\beta=1$.

\subsection{Moments}

The $r$ th moment of $X$ is given by

$$
\begin{aligned}
\mathrm{E}\left(X^{r}\right) & =2 \alpha \beta^{\alpha} \int_{\beta}^{\infty} \frac{x^{r+\alpha-1}}{\left(x^{\alpha}+\beta^{\alpha}\right)^{2}} \mathrm{~d} x \\
& =\frac{2 \alpha \beta^{r}{ }_{2} F_{1}\left(2,2-\frac{r+\alpha}{\alpha} ; 3-\frac{r+\alpha}{\alpha} ;-1\right)}{\alpha-r}, \quad 0<r<\alpha, \beta^{\alpha} \neq 0,
\end{aligned}
$$


where ${ }_{2} F_{1}(\cdot, \cdot ; \cdot ; \cdot)$ is the hypergeometric function; Gradshteyn and Ryzhik [7]. Evidently, Equation (3) involves computation of the confluent hypergeometric function, and routines for this are widely available, such as the hypergeom $(\cdot)$ in MAPLE.

\section{Lorenz curve and Gini index}

The Lorenz curves are commonly used in applied works in areas such as economics, reliability, demography, insurance, medicine and others. For the NP distribution, given $0<p<1$, the Lorenz curve is given by

$$
L(p)=\frac{\int_{0}^{p} q(t) \mathrm{d} t}{\int_{0}^{1} q(t) \mathrm{d} t}=\frac{\int_{0}^{p}\left(\frac{1+t}{1-t}\right)^{1 / \alpha} \mathrm{d} t}{\frac{\alpha}{\alpha-1}{ }_{2} F_{1}\left(1,-\frac{1}{\alpha} ; 2-\frac{1}{\alpha} ;-1\right)}, \quad \alpha>1 .
$$

The Gini index for the NP distribution is given by

$$
\begin{aligned}
\text { Gini } & =1-2 \int_{0}^{1} L(p) \mathrm{d} p=1-2 \int_{0}^{1} \frac{\int_{0}^{p}\left(\frac{1+t}{1-t}\right)^{1 / \alpha} \mathrm{d} t}{\frac{\alpha}{\alpha-1}{ }_{2} F_{1}\left(1,-\frac{1}{\alpha} ; 2-\frac{1}{\alpha} ;-1\right)} \mathrm{d} p \\
& =1-\frac{2}{\frac{\alpha}{\alpha-1}{ }_{2} F_{1}\left(1,-\frac{1}{\alpha} ; 2-\frac{1}{\alpha} ;-1\right)} \int_{0}^{1} \int_{0}^{p}\left(\frac{1+t}{1-t}\right)^{1 / \alpha} \mathrm{d} t \mathrm{~d} p .
\end{aligned}
$$

This integral does not possess a closed-form expression. However, it can be computed numerically by using a software such as $\mathrm{R}$. The $\mathrm{R}$ is freely distributed and available at http://www.r-project.org. In Appendix, we provide a R script to calculate the Gini index from (4).

\section{Maximum likelihood estimation}

We here discuss the ML estimation of the model parameters. Let $x_{(1)} \leq x_{(2)} \leq \cdots \leq x_{(n)}$ be the order statistics for a random sample $X_{1}, X_{2}, \ldots, X_{n}$ of size $n$ from the $\mathrm{NP}(\alpha, \beta)$ distribution. Then, the log-likelihood function can be written as

$$
\ell(\boldsymbol{\theta})=n \log (2 \alpha)-n \log (\beta)+(\alpha+1) \sum_{i=1}^{n} \log \left(\frac{\beta}{x_{i}}\right)-2 \sum_{i=1}^{n} \log \left[1+\left(\frac{\beta}{x_{i}}\right)^{\alpha}\right]
$$

where $\boldsymbol{\theta}=(\alpha, \beta)^{\top}$. It can be seen that $\ell(\alpha, \beta)$ is monotonically increasing with $\beta$, that is, the greater the value of $\beta$, the greater the value of the likelihood function. Hence, since $x \geq \beta$, we conclude that $\widehat{\beta}=x_{(1)}$. The estimator $\widehat{\beta}$ has PDF

$$
z\left(x_{1}\right)=\frac{2^{n} \alpha n \beta^{\alpha n} x_{1}^{\alpha-1}}{\left(x_{1}^{\alpha}+\beta^{\alpha}\right)^{n+1}} \quad x_{1} \geq \beta .
$$


Consequently, the MLE $\widehat{\alpha}$ of $\alpha$ is obtained as the solution to the following equation

$$
\frac{\partial \ell\left(\alpha, x_{(1)}\right)}{\partial \alpha}=-2 \sum_{i=2}^{n} \frac{\left(\frac{x_{(1)}}{x_{(i)}}\right)^{\alpha} \log \left[\frac{x_{(1)}}{x_{i}}\right]}{1+\left(\frac{x_{(1)}}{x_{i}}\right)^{\alpha}}+\sum_{i=2}^{n} \log \left[\frac{x_{(1)}}{x_{i}}\right]+\frac{n}{\alpha}=0 .
$$

\section{Simulation study}

We now carry out a simulation study for evaluating the behavior of the ML estimators. The simulation scenario considers the sample sizes $n \in\{10,50,100\}$, the values of the shape parameter as $\alpha \in$ $\{0.50,0.75,1.00,2.00,4.00,6.00\}, 10,000 \mathrm{MC}$ replications, and the values of the scale parameter as $\beta \in\{1.00,2.00\}$. The values of the shape parameter $\alpha$ have been chosen in order to study the performance under different forms of the distribution.

NP random number generation can be performed using the inverse transform method. Given a uniform variate $U$ drawn from a $\mathrm{U}(0,1)$ distribution, we obtain the variate $X$, which is NP distributed and is given by

$$
X=\left(\frac{2 \beta^{\alpha}}{U}-\beta^{\alpha}\right)^{\frac{1}{\alpha}}
$$

Table 1 reports for each parameter and sample size, the bias and mean squared error (MSE) of the ML estimators. From Table 1, we note that, as the sample size increases, the ML estimates become more efficient, as expected. Furthermore, we note that as the value of shape parameter $\alpha$ increases, the performance of the estimator of $\beta$ improves. It is also noteworthy that when the value of $\beta$ increases from 1 to 2 , the performance of the estimator of $\beta$ deteriorates. In short, the results show the good performance of the ML estimators.

\section{Application to real data}

In this section, we illustrate the proposed NP distribution by analyzing seven real data sets, with two of them taken from the reliability literature and the others corresponding to income data. We compare the results obtained for the NP model, in terms of model fitting, with the corresponding results based on the Pareto, Singh-Maddala and Dagum distributions. In particular, the latter two distributions provide a very good fit to income data and have been widely used to model this kind of data; see McDonald [11]. For each data set, we estimate the unknown parameters of each distribution by the ML method (as discussed in Section 5) and all the computations are carried out using the R software.

\subsection{Reliability data}

The first data set (Set I) is given by Linhart and Zucchini [9], which represents the failure times of the air conditioning system of an airplane: $23,261,87,7,120,14,62,47,225,71,246,21,42,20,5$, $12,120,11,3,14,71,11,14,11,16,90,1,16,52,95$. As a second application, we shall analyze a data set (Set II) from Murthy et al. [13] consisting of the failure times of 20 mechanical components. The data are: $0.067,0.068,0.076,0.081,0.084,0.085,0.085,0.086,0.089,0.098,0.098,0.114$, 
Table 1: Simulated values of biases (MSEs within parentheses) of the estimators of $\alpha$ and $\beta$ of the NP distribution.

\begin{tabular}{ccccccc}
\hline & & \multicolumn{2}{c}{$\mathrm{NP}(\alpha, \beta=1.0)$} & & \multicolumn{2}{c}{$\mathrm{NP}(\alpha, \beta=2.0)$} \\
\cline { 3 - 4 } \cline { 6 - 7 } & $\alpha$ & $\widehat{\alpha}$ & $\widehat{\beta}$ & & $\widehat{\alpha}$ & $\widehat{\beta}$ \\
\hline 10 & 0.50 & $0.0697(0.0376)$ & $0.5654(1.0083)$ & & $0.0692(0.0390)$ & $1.0791(3.3439)$ \\
& 0.75 & $0.1014(0.0835)$ & $0.3237(0.2668)$ & & $0.1001(0.0848)$ & $0.6367(1.0029)$ \\
& 1.00 & $0.1423(0.1539)$ & $0.2216(0.1095)$ & & $0.1382(0.1549)$ & $0.4352(0.4294)$ \\
& 2.00 & $0.2851(0.6170)$ & $0.1002(0.0205)$ & & $0.2549(0.5871)$ & $0.1989(0.0788)$ \\
& 4.00 & $0.5658(2.4430)$ & $0.0479(0.0045)$ & & $0.5266(2.3881)$ & $0.0958(0.0179)$ \\
& 6.00 & $0.8131(5.5365)$ & $0.0314(0.0019)$ & & $0.8522(5.8090)$ & $0.0628(0.0075)$ \\
50 & 0.50 & $0.0115(0.0041)$ & $0.0857(0.0159)$ & & $0.0125(0.0042)$ & $0.1706(0.0634)$ \\
& 0.75 & $0.0200(0.0093)$ & $0.0546(0.0062)$ & & $0.0179(0.0093)$ & $0.1093(0.0245)$ \\
& 1.00 & $0.0260(0.0166)$ & $0.0404(0.0033)$ & & $0.0214(0.0160)$ & $0.0818(0.0137)$ \\
& 2.00 & $0.0490(0.0659)$ & $0.0198(0.0008)$ & & $0.0519(0.0666)$ & $0.0407(0.0033)$ \\
& 4.00 & $0.0952(0.2587)$ & $0.0098(0.0002)$ & & $0.0935(0.2612)$ & $0.0197(0.0008)$ \\
& 6.00 & $0.1304(0.5796)$ & $0.0066(0.0001)$ & & $0.1389(0.5795)$ & $0.0132(0.0003)$ \\
100 & 0.50 & $0.0053(0.0018)$ & $0.0415(0.0036)$ & & $0.0053(0.0019)$ & $0.0812(0.0133)$ \\
& 0.75 & $0.0087(0.0042)$ & $0.0274(0.0015)$ & & $0.0093(0.0042)$ & $0.0537(0.0059)$ \\
& 1.00 & $0.0133(0.0077)$ & $0.0201(0.0008)$ & & $0.0119(0.0076)$ & $0.0402(0.0033)$ \\
& 2.00 & $0.0245(0.0305)$ & $0.0100(0.0002)$ & & $0.0249(0.0308)$ & $0.0204(0.0008)$ \\
& 4.00 & $0.0420(0.1201)$ & $0.0049(<0.0001)$ & & $0.0556(0.1227)$ & $0.0101(0.0002)$ \\
& 6.00 & $0.0870(0.2742)$ & $0.0033(<0.0001)$ & & $0.0728(0.2719)$ & $0.0067(0.0001)$ \\
\hline
\end{tabular}

$0.114,0.115,0.121,0.125,0.131,0.149,0.160$ and 0.485 . Obviously, due to the genesis of the NP distribution, the positive data are by excellence ideally modeled by this distribution. Thus, the use of the NP distribution for fitting these two data sets is well justified.

Table 2 provides some descriptive measures for these two data sets, including central tendency statistics, standard deviation (SD), coefficient of variation (CV), skewness (CS) and kurtosis (CK). From these statistics, we observe the positively skewed nature and moderate (high for the Set II) kurtosis level of the data distributions. The skewed nature is ratified by the histograms of Fig. 3(a) and Fig. 3(d).

Table 2: Descriptive statistics for the data.

\begin{tabular}{cccccccccc}
\hline Set & $n$ & Min. & Median & Mean & Max. & SD & CV & CS & CK \\
\hline Set I & 30 & 1 & 22 & 59.6 & 261 & 71.88 & $120.61 \%$ & 1.61 & 1.64 \\
Set II & 20 & 0.067 & 0.098 & 0.122 & 0.485 & 0.089 & $73.503 \%$ & 3.32 & 10.721 \\
\hline
\end{tabular}

An important characteristic to decide whether a particular distribution is suitable or not for a data set is the shape of a HR. In this sense, we can use the scaled total time on test (TTT) function to detect the type of HR that the data have and then choose a suitable distribution; see Aarset [1]. The TTT plots for the two data sets are presented in Fig. 3(b) and Fig. 3(e), respectively. The TTT plot for the Set I, which represents the failure times of the air conditioning system of an airplane, indicates a decreasing HR, whereas the TTT plot for the Set II, corresponding to the failure times of mechanical components, reveals an upside-down bathtub HR. Therefore, these plots indicate the appropriateness of the NP distribution to fit these data sets, since the new model can present decreasing and upsidedown bathtub (unimodal) HR. 


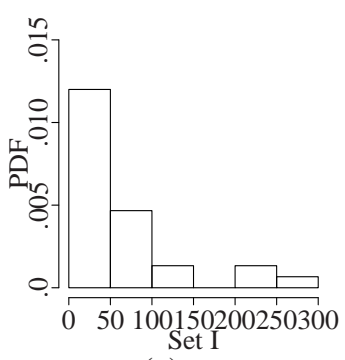

(a)

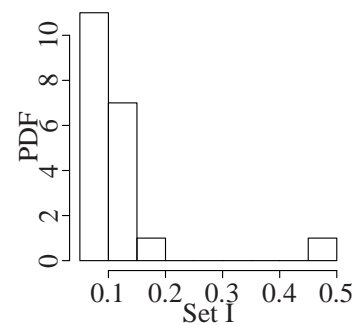

(d)

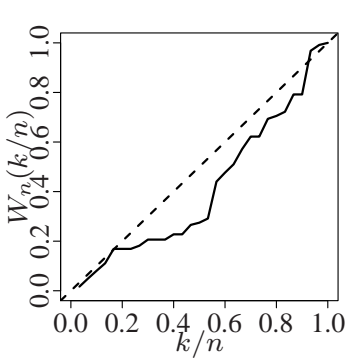

(b)

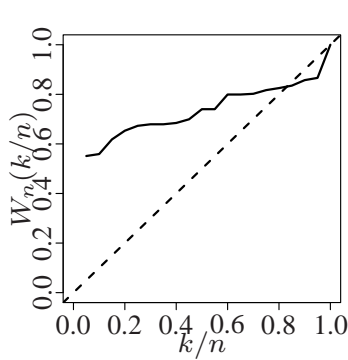

(e)

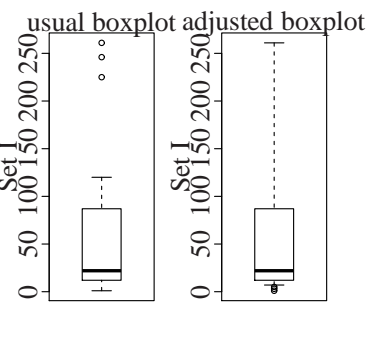

(c)

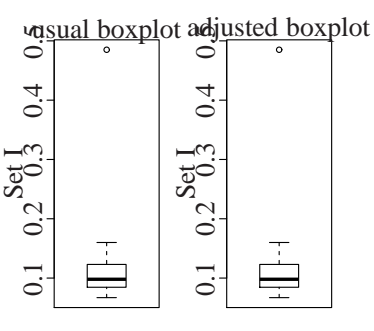

(f)

Figure 3: histogram, TTT plot and boxplots for the Set I (top) and Set II (bottom).

Fig. 3(c) and Fig. 3(f) show the usual and adjusted boxplots, where the latter is useful in cases where the data are skewed distributed, since a significant number of observations can be classified as atypical when they are not; see Hubert and Vandervieren [8]. From Fig. 3(c), we note that potential outliers considered by the usual boxplot are not influential cases when we observe the adjusted boxplot.

Tables 3 reports the ML estimates of the unknown parameters by assuming NP, Pareto, SinghMaddala and Dagum models, respectively, for the Sets I and II. From Table 3, for the Set I and NP model, we note that $\widehat{\alpha} \leq 1$, which implies that the HR of this distribution is decreasing, which is in accordance with Figure 3(b). Furthermore, for the Set II and NP model, $\widehat{\alpha}>1$, implying that the HR is upside-down bathtub, which is also in accordance with Figure 3(e).

Table 3: Maximum likelihood estimates and goodness-of-fit measures.

\begin{tabular}{lcccccccc}
\hline Set & & \multicolumn{3}{c}{ Estimates } & & \multicolumn{3}{c}{ Goodness-of-fit } \\
\cline { 3 - 4 } \cline { 8 - 9 } & Model & $\alpha$ & $\beta$ & $\gamma$ & & AIC & BIC & CAIC \\
\hline \multirow{2}{*}{ Set I } & New Pareto & 0.433 & 1.000 & & & 329.7 & 332.5 & 330.1 \\
& Pareto & 0.298 & 1.000 & & & 338.2 & 341.0 & 338.6 \\
& Singh-Maddala & 0.979 & 170.784 & 3.773 & & 309.7 & 313.9 & 310.6 \\
& $\quad$ Dagum & 1.317 & 30.526 & 0.966 & & 310.4 & 314.6 & 311.3 \\
& & & & & & & & \\
\multirow{4}{*}{ Set II } & New Pareto & 2.871 & 0.067 & & & -76.1 & -74.1 & -75.4 \\
& $\quad$ Pareto & 2.113 & 0.067 & & & -75.1 & -73.1 & -74.4 \\
& Singh-Maddala & 14.331 & 0.075 & 0.187 & & -72.0 & -69.0 & -70.5 \\
& $\quad$ Dagum & 3.784 & 0.031 & 56.361 & & -71.2 & -68.2 & -69.7 \\
\hline
\end{tabular}

The Akaike information criterion (AIC), Bayesian information criterion (BIC) and consistent Akaike information criterion (CAIC) for the NP, Pareto, Singh-Maddala and Dagum models are listed in Table 3. In general, it is expected that the better model to fit the data presents the smaller values 
for these quantities. In terms of the AIC, BIC and CAIC values, the new model seems to be a very competitive model for these two data sets. From this table, we conclude that the NP model provides a better fit to these data than the Pareto, Singh-Maddala and Dagum models for the second data set, and the Singh-Maddala model being better for the first data set.

\subsection{Income data}

We here check whether the NP distribution offers a better fit than the original Pareto, Singh-Maddala and Dagum distributions, to aggregate income data. To do that, the monthly Brazilian GDP (Gross Domestic Product) were obtained for 2002 and 2004. Trying to provide a good proxy for overall per capita income, monthly GDP data were divided per economically active population. The data were obtained from the Central Bank of Brazil and are available at https : / /www3.bcb.gov.br/.

Table 4 provides some descriptive measures for the Brazilian GDP per capita. We observe the relatively low magnitude of skewness and small values of kurtosis of the data distribution.

Table 4: Descriptive statistics for the data.

\begin{tabular}{cccccccccc}
\hline Year & $n$ & Min. & Median & Mean & Max. & SD & CV & CS & CK \\
\hline 2002 & 12 & 5943.592 & 6333.260 & 6389.904 & 6951.542 & 296.421 & 4.639 & 0.364 & -1.004 \\
2004 & 12 & 6760.838 & 7640.401 & 7578.934 & 8285.222 & 468.518 & 6.182 & -0.269 & -1.187 \\
\hline
\end{tabular}

Table 5: Maximum likelihood estimates and goodness-of-fit measures.

\begin{tabular}{lcccccccc}
\hline Year & & \multicolumn{3}{c}{ Estimates } & & \multicolumn{3}{c}{ Goodness-of-fit } \\
\cline { 3 - 4 } \cline { 7 - 8 } & Model & $\alpha$ & $\beta$ & $\gamma$ & & AIC & BIC & CAIC \\
\hline \multirow{2}{*}{2002} & New Pareto & 18.796 & 5943.592 & & & 173.2 & 174.1 & 174.5 \\
& Pareto & 14.000 & 5943.592 & & & 174.9 & 175.9 & 176.3 \\
& Singh-Maddala & 80.115 & 209.826 & 0.004 & & 269.8 & 271.2 & 272.8 \\
& Dagum & 0.919 & 41.362 & 86.618 & & 242.8 & 244.2 & 245.8 \\
& & & & & & & \\
2004 & New Pareto & 12.031 & 6760.838 & & & 187.7 & 188.6 & 189.0 \\
& Pareto & 8.893 & 6760.838 & & & 189.9 & 190.9 & 191.2 \\
& Singh-Maddala & 78.558 & 25.729 & 0.002 & & 286.1 & 287.5 & 289.1 \\
& Dagum & 1.000 & 84.905 & 78.052 & & 244.7 & 246.2 & 247.7 \\
\hline
\end{tabular}

As we have done in previous subsection, the values of the information selection criteria for each model are listed in Table 5. In particular, note that the NP distribution outperforms all the other distributions, suggesting the potentiality of the new model to fit income data.

\subsection{Personal income data}

We now investigate the utility of our model with personal income data. To achieve this goal, we use median annual personal income data (by state) in the United States for 2012 and 2013. The data are available at http://www. census.gov/. In order to remove inflation effects, the database was adjusted to 2014 prices using CPI-U-RS. Some descriptive statistics for these time periods are reported in Table 6. 
Table 6: Descriptive statistics for the data.

\begin{tabular}{cccccccccc}
\hline Year & $n$ & Min. & Median & Mean & Max. & SD & CV & CS & CK \\
\hline 2012 & 52 & 37782 & 52088.5 & 53606.52 & 74072 & 8521.28 & 15.89 & 0.414 & -0.612 \\
2013 & 52 & 32867 & 54374.5 & 55190.10 & 73658 & 9355.48 & 16.95 & 0.059 & -0.800 \\
\hline
\end{tabular}

The MLEs of the parameters and the values of the AIC, BIC and CAIC statistics are listed in Table 7. The values of these statistics indicate that the NP model yields a better fit to these data than the Pareto, Singh-Maddala and Dagum models, suggesting the potentiality and flexibility of the new model to fit personal income data. To illustrate the use of the $\mathrm{R}$ scripts provided in Appendix, we estimate the Gini index for the personal income data. The estimates for 2012 and 2013 are 0.16 and 0.24 , respectively.

Table 7: Maximum likelihood estimates and goodness-of-fit measures.

\begin{tabular}{ccccccccc}
\hline Year & & \multicolumn{3}{c}{ Estimates } & & \multicolumn{3}{c}{ Goodness-of-fit } \\
\cline { 3 - 4 } \cline { 7 - 9 } & Model & $\alpha$ & $\beta$ & $\gamma$ & & AIC & BIC & CAIC \\
\hline \multirow{2}{*}{2012} & New Pareto & 4.304 & 37782 & & & 1113.3 & 1117.2 & 1113.5 \\
& Pareto & 2.962 & 37782 & & & 1126.3 & 1130.2 & 1126.6 \\
& Singh-Maddala & 61.714 & 41704.744 & 0.068 & & 1116.2 & 1122.1 & 1116.6 \\
& Dagum & 0.647 & 23.442 & 96.986 & & 1295.5 & 1301.3 & 1296.0 \\
& & & & & & & & \\
2013 & New Pareto & 2.943 & 32867 & & & 1154.6 & 1158.5 & 1154.8 \\
& Pareto & 1.985 & 32867 & & & 1170.7 & 1174.6 & 1171.0 \\
& Singh-Maddala & 11.189 & 13402.257 & 0.047 & & 1283.6 & 1289.4 & 1284.1 \\
& Dagum & 0.550 & 7.374 & 114.302 & & 1308.3 & 1314.2 & 1308.8 \\
\hline
\end{tabular}

\subsection{Total family income data}

Finally, we consider total family income data taken from the Panel Study of Income Dynamics (PSID), which is a longitudinal household survey in the United States. The income data were collected in 2013 about tax year 2012. The negative values as well as the zeros were removed from the sample. The data and more details about the survey are available at https://psidonline.isr.umich.edu/. We provide some descriptive statistics for these data in Table 8.

Table 8: Descriptive statistics for the data.

\begin{tabular}{cccccccccc}
\hline Year & $n$ & Min. & Median & Mean & Max. & SD & CV & CS & CK \\
\hline 2012 & 24749 & 3 & 55000 & 75389.63 & 6301000 & 130329.4 & 172.87 & 25.71 & 1013.71 \\
\hline
\end{tabular}

The MLEs of the parameters and the values of the AIC, BIC and CAIC statistics are listed in Table 9. The values of these statistics indicate that the Dagum model outperforms all other models. However, the NP model provides a better fit than the Pareto model.

\section{Concluding remarks}

In this work, we have introduced a new generalization of the Pareto distribution. The new model presents more simplicity in mathematical terms and allows the hazard rate to have either upside-down 
Table 9: Maximum likelihood estimates and goodness-of-fit measures.

\begin{tabular}{ccccccccc}
\hline Year & & \multicolumn{3}{c}{ Estimates } & & \multicolumn{3}{c}{ Goodness-of-fit } \\
\cline { 3 - 4 } \cline { 7 - 9 } & Model & $\alpha$ & $\beta$ & $\gamma$ & & AIC & BIC & CAIC \\
\hline 2012 & New Pareto & 0.159 & 3 & & & 686117.2 & 686133.5 & 686117.3 \\
& Pareto & 0.103 & 3 & & & 695640.1 & 695656.3 & 695640.1 \\
& Singh-Maddala & 4.189 & 1829.434 & 0.068 & & 644873.7 & 644898 & 644873.7 \\
& Dagum & 1.371 & 19105.375 & 2.639 & & 607903.7 & 607928.1 & 607903.7 \\
\hline
\end{tabular}

bathtub or decreasing shape, depending on the values of its parameters. A Monte Carlo simulation study was carried out to evaluate the behavior of the proposed model parameters. We have illustrated the usefulness of the proposed model by its good fitting in the application. We hope that the new distribution may serve as an alternative distribution to other models available in the literature for modeling real data in areas such as engineering, biology, finance, hydrology, economics, among others.

\section{Acknowledgements}

The authors thank the two anonymous referees and an associate editor for the constructive suggestions and comments. We thank the financial support from Conselho Nacional de Desenvolvimento Científico e Tecnológico (CNPq-Brazil) and Coordenação de Aperfeiçoamento de Pessoal de Nível Superior (CAPES-Brazil). The collection of PSID data used in this study was partly supported by the National Institutes of Health under grant number R01 HD069609 and the National Science Foundation under award number 1157698.

\section{Appendix. R Scripts}

The following algorithm calculate the maximum likelihood estimates of the NP model parameters and the Gini index from (4).

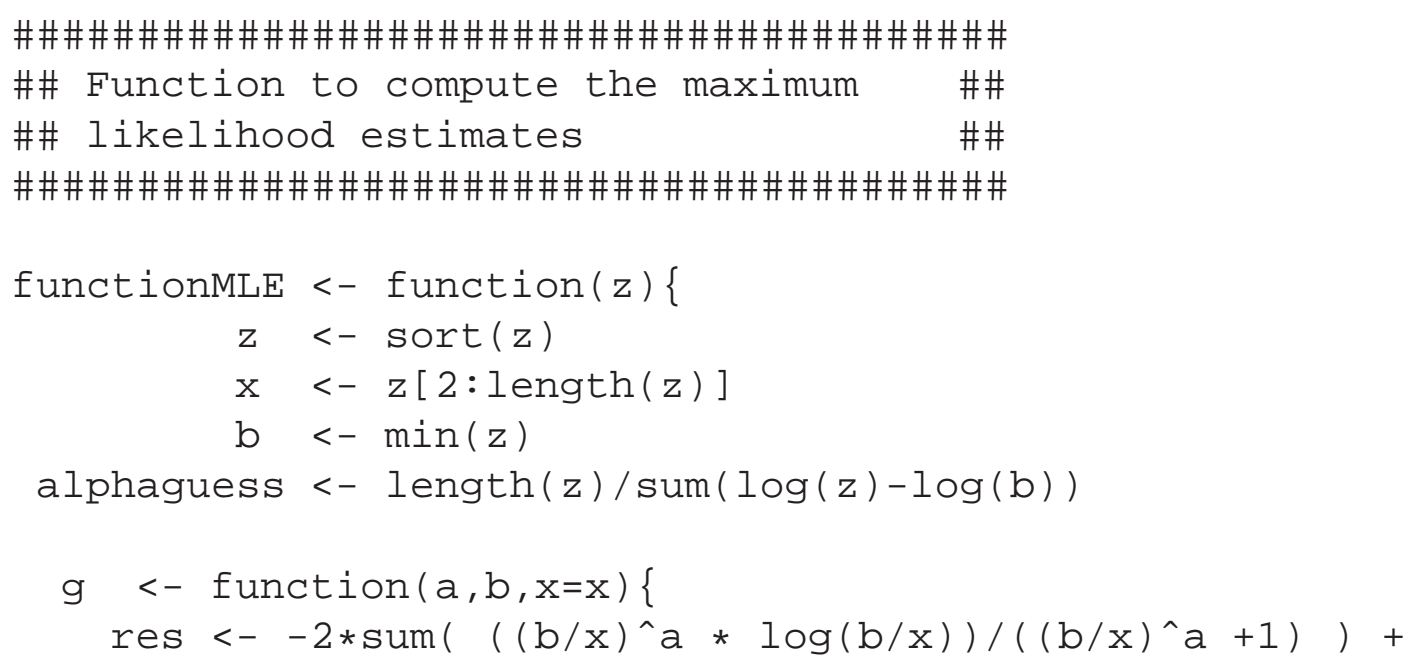




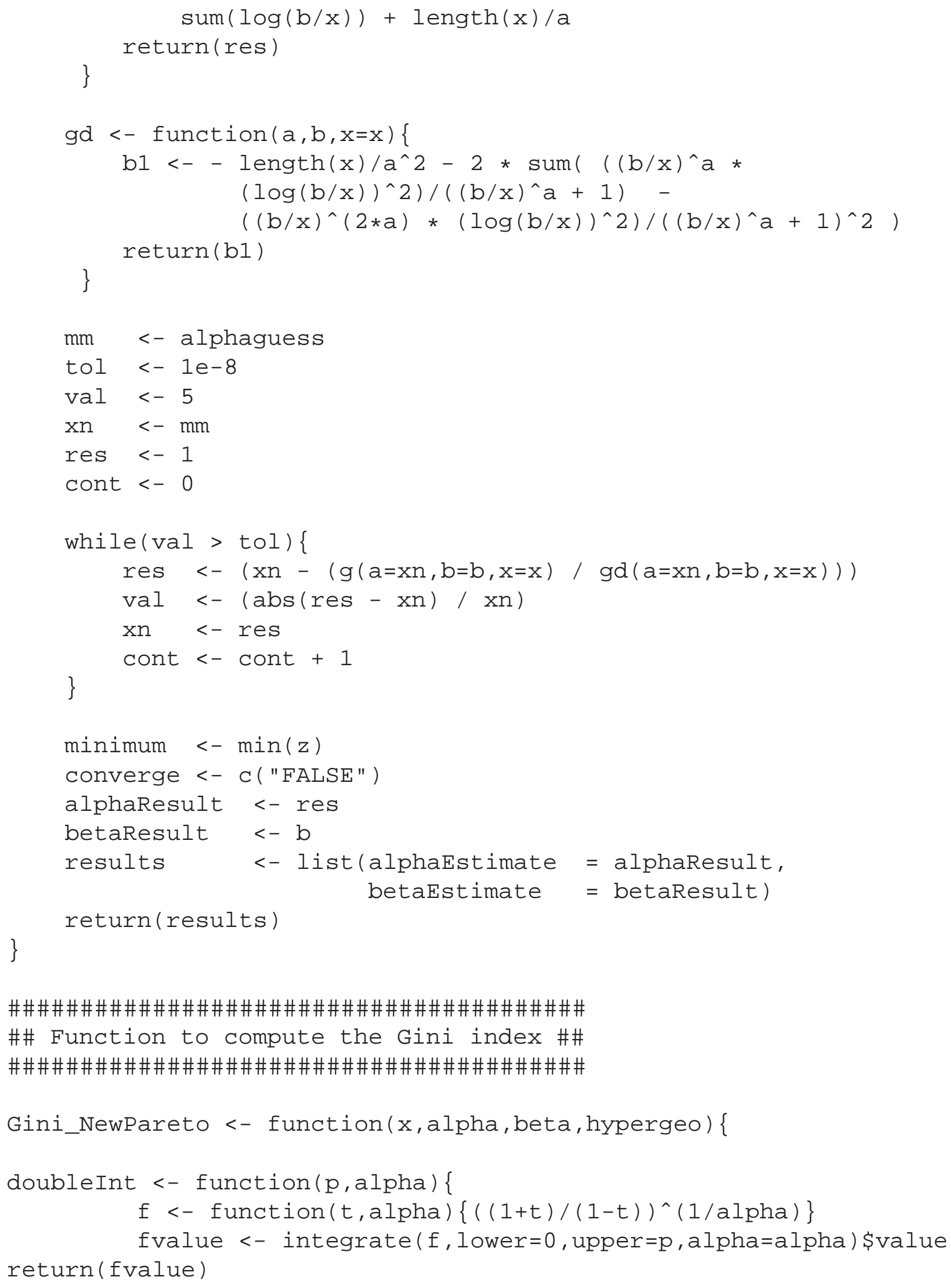




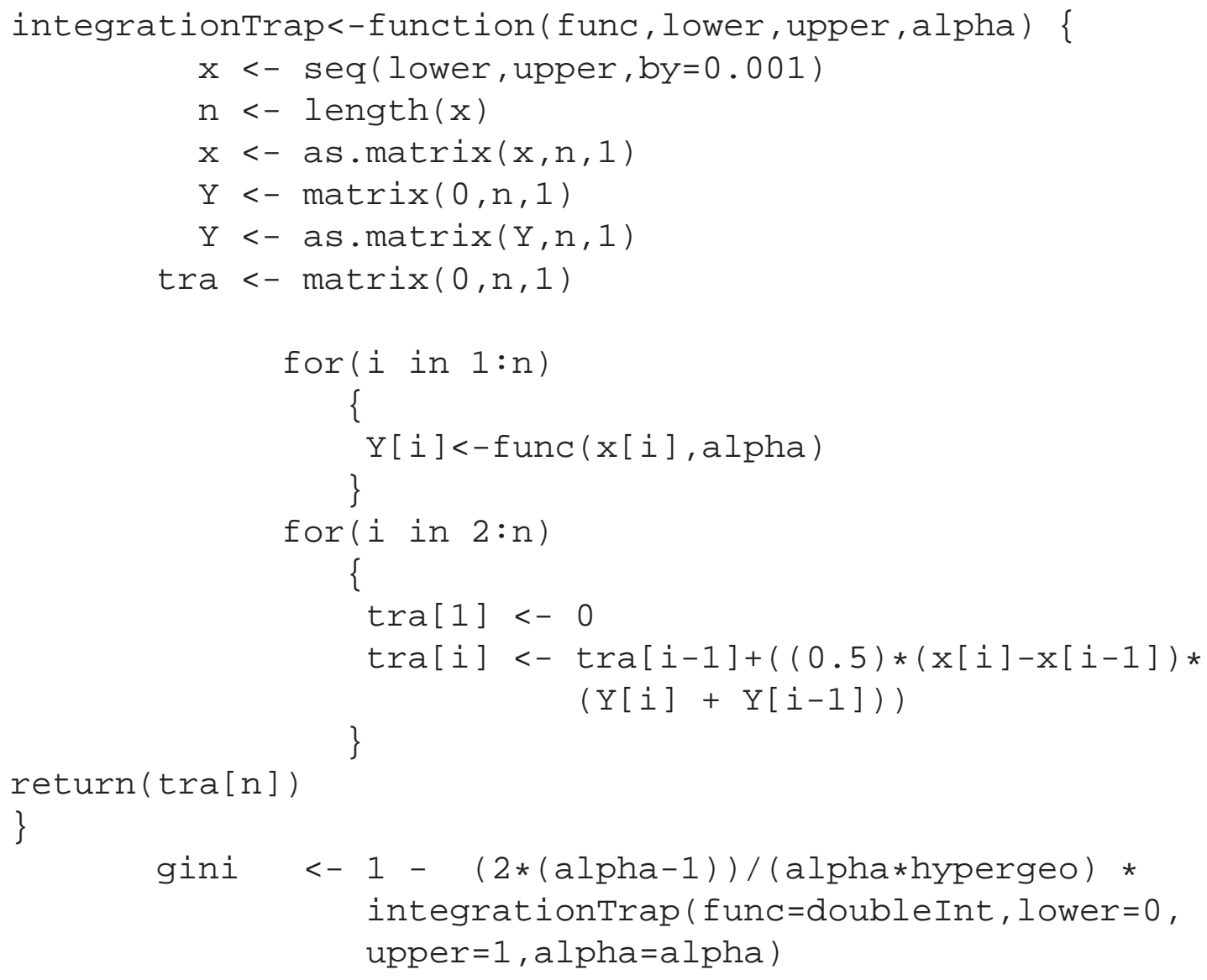

\section{References}

[1] M.V. Aarset, How to identify bathtub hazard rate, IEEE Transactions on Reliability, 36 (1987) 106-108.

[2] F. Clementi,, M. Gallegati, G. Kaniadakis, $\kappa$-generalised statistics in personal income distribution, Eur. Phys. J.B., 57 (2007) 187-193.

[3] F. Clementi, T. Di Matteo,, M. Gallegati, G. Kaniadakis, The $\kappa$-generalised distribution: a new descriptive model for the size distribution of incomes, Physica A, 387 (2008) 3201-3208.

[4] F. Clementi, M. Gallegati, G. Kaniadakis, A $\kappa$-generalized statistical mechanics approach to income analysis, J. Stat. Mech., (2009). arXiv:0902.0075v2.

[5] A. Dragulescu, V.M. Yakovenko, Evidence for the exponential distribution of income in the USA, Eur. Phys. J.B., 20 (2001) 585-5899.

[6] F.C. Figueira, N.J. Moura Jr., M.B. Ribeiro, The Gompertz Pareto income distribution, Physica A, 390 (2010) 689-698. 
[7] I.S. Gradshteyn, I.M. Ryzhik, Tables of Integrals, Series, and Products, Edited by A. Jeffrey and D. Zwillinger, 7th edition, Academic Press, New York, 2007.

[8] M. Hubert, E. Vandervieren, An adjusted boxplot for skewed distributions, Computational Statistics \& Data Analysis, 52 (2008) 5186-5201.

[9] H. Linhart, W. Zucchini, Model Selection, Wiley, New York, 1986.

[10] B. Mandelbrot, The Pareto-Lévy law and the distribution of income, International Economic Review, 1 (1960) 79-106.

[11] McDonald, J.B., Some generalized functions of the size distribution of income, Econometrica, 52 (1984) 647-663.

[12] N.J. Moura Jr., M.B. Ribeiro, Evidence for the Gompertz curve in the income distribution of Brazil 1978-2005, Eur. Phys. J.B., 67 (2009) 101-120.

[13] D.N.P. Murthy, M. Xie, R. Jiang, Weibull models, Wiley series in probability and statistics, Hoboken (NJ): JohnWiley and Sons, 2004.

[14] V. Pareto, Cours dćonomie Politique, Lausanne, 1987.

[15] C.A. Silva, Applications of physics to finance and economics: returns, trading activity and income, Ph.D. Thesis, University of Maryland, 2001.

[16] G. Willis, J. Mimkes, Evidence for the independence of waged and unwaged income, evidence for Boltzmann distributions in waged income, and the outlines of a coherent theory of income distribution, e-print, 2004. arXiv:cond-mat/0406694v1.

[17] V.M. Yakovenko, J.B.Rosser, Colloquium: statistical mechanics of money, wealth, and income, Rev. Modern Phys., 81 (2009) 1703-1725. 